\title{
Belgeo
}

Revue belge de géographie

Delgadillo Macías J., Hildenbrand Scheid A., Garrido Yserte R. (dir.), Planificación regional y ordenación territorial : visiones contemporáneas desde España y México

Madrid, Fondo de cultura económica, 2021

\section{Thomas Perrin}

\section{OpenEdition}

\section{Journals}

Édition électronique

URL : https://journals.openedition.org/belgeo/52289

DOI : 10.4000/belgeo.52289

ISSN : 2294-9135

Éditeur :

National Committee of Geography of Belgium, Société Royale Belge de Géographie

\section{Référence électronique}

Thomas Perrin, « Delgadillo Macías J., Hildenbrand Scheid A., Garrido Yserte R. (dir.), Planificación regional y ordenación territorial : visiones contemporáneas desde España y México », Belgeo [En ligne], 3 | 2021, mis en ligne le 18 février 2022, consulté le 06 avril 2022. URL : http://journals.openedition.org/ belgeo/52289; DOl : https://doi.org/10.4000/belgeo.52289

Ce document a été généré automatiquement le 6 avril 2022.

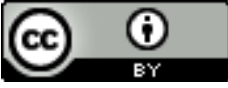

Belgeo est mis à disposition selon les termes de la licence Creative Commons Attribution 4.0 International. 


\section{Delgadillo Macías J.,} Hildenbrand Scheid A., Garrido Yserte R. (dir.), Planificación regional y ordenación territorial : visiones contemporáneas desde España y México

Madrid, Fondo de cultura económica, 2021

Thomas Perrin

\section{RÉFÉRENCE}

Delgadillo Macías J., Hildenbrand Scheid A., Garrido Yserte R. (dir.), 2021, Planificación regional y ordenación territorial : visiones contemporáneas desde España y México, Madrid, Fondo de cultura económica

L'ouvrage Planificación regional y ordenación territorial, coordonné par Javier Delgadillo Macías, Andreas Hildenbrand Scheid et Rubén Garrido Yserte aux éditions Fondo de cultura económica, présente un double intérêt. Tout d'abord il livre une vision documentée et actualisée des politiques et dispositifs d'aménagement du territoire en Espagne, en croisant les spécificités nationales avec les dynamiques d'européanisation à l'œuvre. De plus, l'ouvrage met la situation espagnole en perspective internationale avec les politiques en vigueur au Mexique, un pays certes non européen mais qui partage une certaine proximité historique et culturelle avec l'Espagne. Dans cette logique, l'ouvrage est composé de deux parties : la première porte sur l'Espagne et la seconde sur le Mexique. Les chapitres abordent des considérations plutôt générales sur les systèmes d'aménagement, des cas particuliers et des exemples plus localisés. 
2 La première partie s'ouvre avec un panorama très complet et à visée comparative des politiques développées par les 17 communautés autonomes qui composent l'Espagne depuis la transition démocratique à la fin des années 1970 (Andreas Hildenbrand Scheid). Joaquín Farinós Dasí revient ensuite sur le concept même d'aménagement du territoire pour en révéler la complexité, l'« intégralité et la transversalité ", à la lumière notamment des objectifs et des critères de la politique de cohésion de l'Union européenne. Le chapitre collectif qui suit (Julián Mora Aliseda, Jacinto Garrido Velarde, Manuel Díaz González) s'intéresse aux déclinaisons sectorielles des politiques de planification, qui requièrent un grand effort de coopération et de coordination entre différents niveaux décisionnels en fonction des secteurs: politiques étatiques, régionales, locales. Un constat que l'on peut faire de tout système d'aménagement dans les sociétés complexes européennes. Les deux chapitres suivants (Juan Raposo Arceo, José Antonio Segrelles) resserrent la focale sur des études de cas dans des régions particulières: articulation entre planification régionale et urbaine dans le plan d'aménagement du littoral en Galice, création d'un parc naturel dans la Communauté valencienne. Enfin, la première partie se termine avec une réflexion critique sur l'application en Espagne de la politique de cohésion de l'Union européenne, dont les injonctions peuvent se révéler contradictoires (Tomás Mancha Navarro, Rubén Garrido Yserte, María Teresa Gallo Rivera).

3 La partie sur le Mexique suit le même cheminement logique, avec d'abord deux chapitres qui posent le contexte à travers l'évolution des politiques territoriales dans le pays. José Gasca Zamora met en lumière les continuités et ruptures de cette évolution, ainsi que l'impact sur l'environnement et le développement social qu'ont pu produire les différentes politiques suivies, dans un pays où le taux de pauvreté et les inégalités restent relativement élevées notamment dans certaines régions. Adrián Guillermo Aguilar Martinez revient en détail sur le contenu et le déploiement de la loi nationale sur l'aménagement et l'urbanisme adoptée en 2016, qui d'après lui constitue, par de nombreux aspects, un rendez-vous manqué. À la suite de cela, Mario Miguel Carrillo Huerta présente des instruments de l'aménagement régional, avec des exemples concrets de mise en œuvre à des échelles originales, microrégions ou mesorégions, qu'il soumet à une analyse critique. Le chapitre de Felipe Torres Torres propose des pistes de rénovation des politiques d'aménagement et de développement durable des territoires dans le contexte mexicain, tandis que Javier Deladillo Macías clôt cette partie avec différentes expériences concrètes qui illustrent tant les problèmes que les succès de ces politiques.

4 Dès la présentation le propos est dense, précis. L'ouvrage fait le point sur les nuances entre les différents "domaines" et les échelles variées des politiques territoriales, entre planification, aménagement, urbanisme; entre régions, villes, agglomérations urbaines; zones rurales ou métropoles. Les auteurs proposent à la fois des réflexions de fond, des exemples concrets et variés, des exposés plus techniques ou qui touchent à la mise en œuvre concrète des politiques. L'ouvrage remplit ainsi l'objectif ambitieux de croiser théorie et pratique, et ce dans une perspective comparée originale. Il fournit ainsi des informations utiles pour envisager des projets et des collaborations scientifiques à l'échelle européenne et internationale. L'ouvrage intéressera un lectorat large : chercheur, étudiant, praticien, responsable politique, journaliste par exemple. Le lecteur français remarquera la mention d'une spécificité du modèle d'aménagement du territoire en France par rapport à d'autres façons de faire en Europe. La perspective 
européenne est d'ailleurs particulièrement éclairante à suivre dans le cas de l'Espagne, car d'une part elle montre que de nombreuses problématiques d'aménagement sont communes aux pays européens et elle confirme, d'autre part, l'européanisation croissante des politiques et des programmes d'aménagement. L'ouvrage permet aussi de déconstruire certaines visions, comme le fait que le Mexique, pays fédéral, a des politiques d'aménagement parfois plus centralisées que l'Espagne, non officiellement une fédération mais dont le modèle original "d'état des autonomies " laisse une très large marge de manœuvre aux régions notamment dans le domaine de l'aménagement. On observe ainsi des nuances entre les cultures d'aménagement des différentes communautés autonomes qui composent l'Espagne, dont certaines font parfois de leurs politiques territoriales un instrument de différenciation. Enfin, l'ouvrage confirme la place particulière et affirmée de l'échelle régionale dans l'aménagement des territoires et le développement des sociétés, comme le montre une série de publications récentes ou à paraître (Perrin, Seys, 2019a, 2019b ; Harrison, Galland \& Tewdwr-Jones, 2021 ; Purkarthofer, Humer \& Mäntysalo, 2021 ; Neuman, Zonneveld, 2021; Perrin, 2022a, 2022b).

Si la densité de l'ouvrage de plus de 450 pages peut parfois rendre la lecture plus ardue, en particulier une lecture continue, l'épaisseur analytique et informationnelle des chapitres, ou de groupes de chapitres, peut permettre de les considérer assez indépendamment, à la manière d'un manuel ou d'un compendium, mobilisable pour différents types de travaux en fonction des thématiques visées. Le propos aurait pu gagner en efficacité avec plus d'illustrations, de schémas ou tableaux de synthèse, même si de tels visuels sont présents dans plusieurs chapitres comme par exemple les tableaux en pages 62 et 64, bienvenus pour étayer l'exposé. Si tant est que la lecture de l'espagnol ne soit pas une barrière, cet ouvrage est une référence incontournable sur l'aménagement régional contemporain, à la fois sur un plan général et sur les cas particuliers de l'Espagne et du Mexique. Il sera un outil précieux pour tout lecteur d'espagnol qui travaille sur le contexte européen et international.

\section{BIBLIOGRAPHIE}

HARRISON J., GALLAND D. \& TEWDWR-JONES M. (dir.) (2021), Planning regional futures, Regional Studies, $55,1$.

NEUMAN M., ZONNEVELD W. (dir.) (2021), The Routledge Handbook of Regional Design, Abingdon, Routledge.

9 PERRIN T., SEYS F.-O. (dir.) (2019a), La région, vous dîtes? Le kaléidoscope régional de l'Union européenne, Belgeo, 2, https://doi.org/10.4000/belgeo.28463

PERRIN T., SEYS F.-O. (dir.) (2019b), Régions en tension, régions en recomposition. Le SudOuest européen en perspective, Sud-Ouest Européen, 48.

PERRIN T. (2022a), La région au XXI e siècle. Perspectives de France et d'Europe, Bruxelles, PIE-Peter Lang.

PERRIN T. (dir.) (2022b), Regions and regional planning: Experiences from France and Europe, Abingdon, Routledge.

PURKARTHOFER E., HUMER A. \& MÄNTYSALO R. (dir.) (2021), Regional planning: interests, institutions and relations, Regional Studies, 55, 5. 